\title{
The ubiquitous 'cancer mutational signature' 5 occurs specifically in cancers with deleted FHIT alleles
}

\author{
Stefano Volinia ${ }^{1}$, Teresa Druck², Carolyn A. Paisie ${ }^{3}$, Morgan S. Schrock ${ }^{2}$ and Kay \\ Huebner ${ }^{2}$ \\ ${ }^{1}$ Department of Morphology, Surgery \& Experimental Medicine, University of Ferrara, 44121 Ferrara, Italy \\ ${ }^{2}$ Department of Cancer Biology \& Genetics, The Ohio State University Comprehensive Cancer Center \& Wexner Medical \\ Center, Biomedical Research Tower, Columbus, OH 43210, USA \\ ${ }^{3}$ University of Washington, Department of Biomedical Informatics \& Medical Education, Center for Infectious Disease \\ Research, Seattle, WA 98109, USA \\ Correspondence to: Kay Huebner, email: kay.huebner@osumc.edu \\ Keywords: exome sequences; mutational signatures; data mining; clock-like signatures; cancer genes \\ Received: September 07, $2017 \quad$ Accepted: October 25, $2017 \quad$ Published: November 06, 2017 \\ Copyright: Volinia et al. This is an open-access article distributed under the terms of the Creative Commons Attribution License \\ 3.0 (CC BY 3.0), which permits unrestricted use, distribution, and reproduction in any medium, provided the original author and \\ source are credited.
}

\section{ABSTRACT}

The FHIT gene is located at the fragile FRA3B locus where activation by carcinogen-induced and endogenous replication stress causes FHIT deletions even in normal cells over a lifetime. Our lab has shown that loss of FHIT expression causes genome instability and provides single-strand DNA substrates for APOBEC3B hypermutation, in line with evidence that FHIT locus deletions occur in many cancers. Based on these biological features, we hypothesized that FHIT loss drives development of COSMIC mutational signature 5 and here provide evidence, including data mining of $>6,500$ TCGA samples, that FHIT is the cancer-associated gene with copy number alterations correlating most significantly with signature 5 mutation rate. In addition, tissues of Fhit-deficient mice exhibit a mutational signature strongly resembling signature 5 (cosine similarity value $=0.89$ ). We conclude that FHIT loss is a molecular determinant for signature 5 mutations, which occur in all cancer types early in cancer development, are clock-like, and accelerated by carcinogen exposure. Loss of FHIT caretaker function may be a predictive and preventive marker for cancer development.

\section{INTRODUCTION}

Frequent deletions within the fragile FRA3B/FHIT locus in preneoplasias [1-6], leading to loss or reduction of Fhit protein expression, are due to the sensitivity of this common fragile site to replication stress. In normal, transformed, and cancer-derived cell lines, Fhit-depletion causes replication stress-induced DNA double-strand breaks $[7,8]$ and defects in replication fork progression, through down-regulation of Thymidine Kinase 1 (TK1) expression and reduced thymidine triphosphate pool levels; thymidine supplementation rescues DNA replication defects and suppresses DNA breakage in Fhitdeficient cells. Depletion of Fhit does not activate the DNA damage response, allowing continued cell proliferation and ongoing chromosomal instability [7]. Also, Waters et al [9] showed that FHIT-low/APOBEC3B(A3B)-high cytidine deaminase-expressing lung adenocarcinomas displayed increased numbers of A3B signature mutations, while tumors with normal FHIT expression did not exhibit A3B hypermutation, in spite of high A3B expression; thus, A3B overexpression and Fhit-loss induced DNA damage are independent events that when occurring together, result in increased A3B induced mutations. These biological and genetic features of cells and cancers with reduced FHIT expression, suggested that reduced FHIT expression might drive generation of a specific cancer-associated 'mutational signature' defined by Alexandrov et al [10] as 
Catalog of Somatic Mutations in Cancer (COSMIC; http:// cancer.sanger.ac.uk/cosmic) [11] mutational signature 5.

Using a 96-category single base substitution (SBS) classification, based on type of substitution and bases immediately $5^{\prime}$ and $3^{\prime}$ to the mutated base, Alexandrov \& colleagues have identified 30 distinct mutational signatures across 40 cancer types, accessible in the COSMIC database $[10,11]$. Some signatures, such as signature 5, are present in multiple cancer types, while others are restricted to a certain class of cancer. For instance, signature 7 , which is found primarily in skin cancers and is characterized by the presence of $\mathrm{CC}>\mathrm{TT}$ dinucleotide mutations at dipyrimidines, is believed to be caused by Ultraviolet light [11]. In follow-up studies $[12,13]$, Alexandrov \& coauthors used mutations from $>10,000$ cancer genomes representing 36 cancer types, to investigate clock-like mutational processes in human cells and reported that only two mutational signatures showed clock-like properties, with different mutation rates in different tissues $[12,14]$. Since the mutation rates for the two signatures were not correlated, it was concluded that processes driving signatures $1 \& 5$ throughout life, were different but mutation numbers for both increased in correlation with age. Thus, the set of 'somatic mutations shared by most members of a cancer cell population, is the set that was present in the progenitor cell of the final dominant clonal expansion of the cancer' [14]. Since the FHIT gene encompasses a common fragile site, common to all humans (and mice), the locus accumulates chromosome gaps in some cells and likely most tissues throughout life [15-17]; also age-associated mutation would increase due to loss of genome caretaker function in the cells with FHIT locus gaps/deletions. That is, endogenous replication stress associated with aging results in alterations within the FRA3B locus, loss of FHIT genome caretaker function, imbalance of deoxynucleotide triphosphate pools and enhanced replication stress $[7,8]$. We have thus proposed that loss of FHIT expression underlies development of the ubiquitous signature 5 mutations in human cancers.

\section{RESULTS}

\section{The mutation profile of Fhit knockout mouse genomes resembles COSMIC signature 5}

Allele copy number alterations (CNAs) and expression changes are observed in Fhit-deficient cells in conjunction with alterations in cell proliferation and exome mutations [16, 18-20]. To define genomic changes associated with preneoplastic changes in vivo, exome DNAs were sequenced for mouse Fhit wild-type (wt) and knockout (ko) tissues and established kidney cell lines. The ko exome DNAs showed increased frequencies of small insertions, deletions and SBSs relative to wt DNAs, some related to preneoplastic changes [7, 18-20]. Thus, Fhit loss provided a 'mutator' phenotype, a cellular environment in which mild genome instability permits clonal expansion through proliferative and survival advantage.

As noted in Paisie et al [20], the mutation profile in Fhit ko tissues and cell lines is characterized by increased $\mathrm{C}>\mathrm{T}$ and $\mathrm{T}>\mathrm{C}$ mutations, resembling human mutational signature 5 [10]; compare Figure 1A, upper and middle panels. Alexandrov et al [10], by 2013, had analyzed $>5$ million mutations in $>7000$ cancers, from which they extracted distinct mutational signatures, some of which were observed in many cancer types, including: two signatures, $2 \& 13$, attributed to the APOBEC family of cytidine deaminases; signature 1 associated with patient age at cancer diagnosis; signatures 4, 7, 11 associated with known mutagenic exposures; signatures 3, 6, 10 associated with mutations in DNA repair proteins, known as genome caretakers. The mutation sources for many signatures, including signature 5, were unknown; however signature 5 (Figure 1A middle panel) was recently shown, along with the 'aging' signature, to be associated with sequences of genomes of all 40 cancer types [10, 11 and COSMIC Database http://cancer.sanger.ac.uk/cosmic], 22 of which also showed APOBEC signatures $2 \& 13$. Depictions of signature 5 are illustrated in Figure 1A, middle and lower panels to show their similarity to each other. Figure 1A, lower panel, designated Signature $5^{*}$, was extracted from human bladder cancers by Kim et al [21] using nonnegative matrix factorization (NMF) algorithms similar to those described by Alexandrov and colleagues. Despite subtle differences, the Fhit ko kidney mutation spectrum has a cosine similarity of 0.85 and 0.80 , with COSMIC signature 5 and Kim et al [21] signature $5^{*}$, respectively. A cosine similarity of 0.00 indicates completely different and a similarity of 1.00 indicates a perfect match.

\section{Mutational signatures in Fhit knockout cells and tissues}

To confirm the similarity of the Fhit ko mutation profile to human cancer mutational signature 5 , we used the SomaticSignatures package [22] and extracted two mutational signatures from exome sequences of six mouse Fhit ko samples described in Paisie et al [20] (Figure 1B). Because most tumors contain more than one mutational signature, the SomaticSignatures package enables users to extract a user-defined number of expected signatures from sequencing data, with power to detect signatures increasing with the number of signatures entered into the algorithm. DNA from age-matched wt mice exhibited a range of 19 to $\sim 300 \mathrm{SBSs}$, too few to reliably extract signatures, while Figure 1B depicts two signatures ('Tissue culture signature' and 'Fhit loss signature') that were extracted from six DNA samples obtained from kidney, liver and embryonic stem cells of Fhit -/- mice, all having between 2,596 and 4,757 SBSs; the Fhit ko mouse tissues exhibit elevated numbers of mutations because of 
absence of the Fhit genome caretaker function [20]. The 'tissue culture signature' found in exome sequences of cells subcultured in vitro $>15$ times [20], features a prominent GCC to GGC mutation. This signature was not found in the exomes of the mouse tissues or embryonic stem cell lines (ESC, subcultured in vitro for 1 or 2 passages) examined. The 'Fhit loss mutational signature' occurred in exome DNAs of all Fhit ko mouse samples, both cell lines and tissues. This signature closely resembles the reported mouse kidney FHIT ko mutation profile shown in Figure 1A, upper panel, and has a cosine similarity value of 0.89 with COSMIC signature 5. For comparison, signature 5 from Alexandrov et al [10] is shown in Figure 1A, middle panel (see Supplementary Figure 1 for the individual fitted spectra from the application of the SomaticSignatures package for total SBS graphs for each tissue and cell line). As a guide for the impact of cosine similarity values, we calculated the values for signatures $2(0.38$, an apobec expression signature), 8 ( 0.71 , a loss of mismatch repair signature) and 13 (0.24, another apobec expression signature) for comparison with the 'Fhit loss mutational signature'.
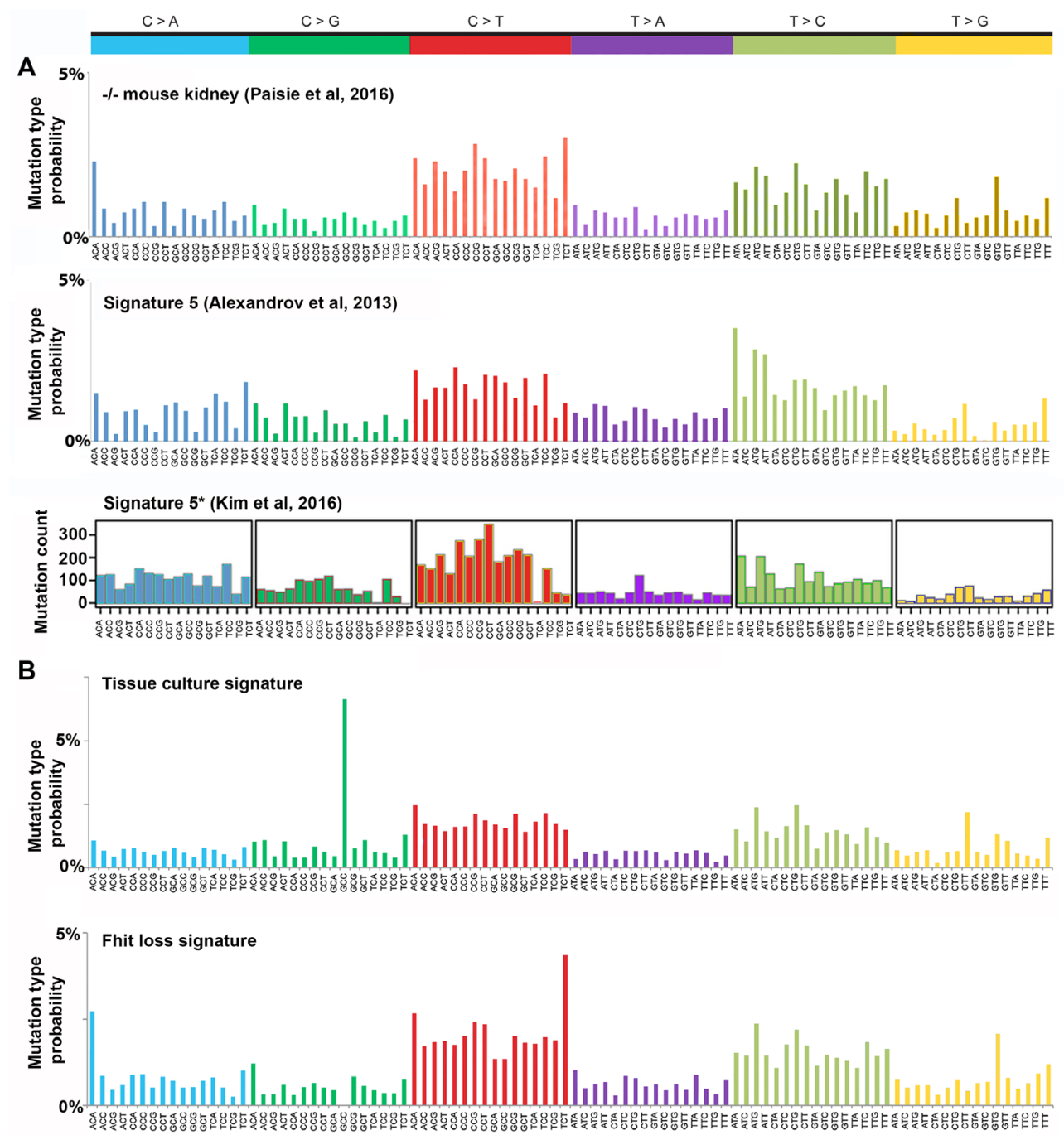

Figure 1: Fhit knockout mutational signature compared to signature 5 profiles. (A) Previously described mutational signatures from Fhit ko mouse kidney tissue total mutation profile adapted from Figure 3 of Paisie et al [20], human mutational signature 5 of Alexandrov et al [10], signature $5^{*}$ from Kim et al [21] that used a different algorithm to define mutational signatures. \{Permissions: upper panel, Paisie et al (2016) under CC BY-NC 4.0 license (https://creativecommons.org/licenses/by-nc/4.0/legalcode); middle panel, adapted by permission from Macmillan Publishers Ltd: [Nature] Alexandrov et al. 2013; lower panel, adapted by permission from Macmillan Publishers Ltd: [Nature Genetics] Kim et al. 2016\}. (B) The mutation spectra for Fhit-/- lung, kidney tissues, two -/- kidney cell lines, a totipotent and differentiated -/- ESC cell line, were assessed for mutational signatures using the SomaticSignatures algorithm [22]. The upper panel (tissue culture signature) shows a signature occurring primarily in the two kidney cell lines, with a distinctive peak of C to $\mathrm{G}$ SBSs at GCC trinucleotides; the lower panel (FHIT loss signature) shows a mutational signature dominated by $\mathrm{C}$ to $\mathrm{T}$ and $\mathrm{T}$ to $\mathrm{C}$ SBSs that may be the murine equivalent of the human signature 5 shown in panel A. 


\section{FHIT loss correlates with the mutational signature 5 substitution rate in human cancer}

To determine whether loss of FHIT, or 86 other cancer-relevant or cancer-driver genes [23, 24] (see Supplementary Table 1 for gene list), may be associated with the reported prevalence of mutational signature 5 in human cancers, we compiled the Somatic Copy Number Alterations (SCNAs) of FHIT and the other 86 cancer genes in The Cancer Genome Atlas (TCGA) samples $(n=6649)$ previously characterized for mutational signatures. Spearman Rho and robust regression MM estimates $[25,26]$ were computed between the signature 5 somatic substitution rate per $\mathrm{Mb}$ [13] and thresholded GISTIC2 score for the selected SCNAs genes, across the TCGA cohorts. Table 1 lists the genes with SCNAs that were negatively or positively associated with mutational signature 5 in the 6649 cancer samples, identifying FHIT as the gene exhibiting the most significant, and negative, correlation with mutational signature 5 (Spearman $\mathrm{p}=$ 2.00E-65, MM Regression $\mathrm{p}=1.90 \mathrm{E}-25)$; i.e., FHIT copy loss (negative GISTIC2 scores) was associated with higher density of signature 5 substitutions in the genomes of cancer cells. Supplementary Table 2 shows substitution rate/Mb [12] vs FHIT allele copy number (thresholded GISTIC2 score) for mutational signature 5 in each TCGA sample. Other fragile site genes included in this study, such as PARK2 and WWOX, did not correlate with signature 5. NAALADL2, the second most significant signature 5-associated gene, exhibited positive correlation, not coherent with its loss leading to higher signature 5 substitutions. A few deleted genes showed significant but weaker negative correlations, viz CSMD1, RYR2, DSCAM, while TP53, ETV6 and MAP2K4, like NAALADL2 showed incoherent trends.

Next, we looked for associations between FHIT loss and other mutational signatures. The number of TCGA cancers assessed for each cancer type was dependent on the occurrence of individual mutational signatures in specific TCGA cancer types; for each signature, a Spearman correlation and MM regression were computed for the comparison of the somatic substitution rate/Mb vs FHIT copy number (Table 2). Only two signatures were significant both by Spearman correlation and robust regression, signatures 5 and 2. Mutational signature 5 was the signature most negatively correlated with FHIT copy number alterations, closely followed by mutational signature 2, caused by activity of enzymes A3B or A3A $(\mathrm{n}=3702$, Rho $\mathrm{p}=5.30 \mathrm{E}-43$, MM $\mathrm{p}=1.1 \mathrm{E}-21)$; A3B mutations were previously shown to be increased in cells with Fhit expression loss [9]. Signature 13, the other A3B/A signature, was also correlated with FHIT SCNAs, though less significantly and not confirmed by the MM regression test.

\section{Genes with mutations or SCNAs associated with FHIT allele loss}

We also assessed allele losses, gains and gene mutations, for association with loss of FHIT in the TCGA cohorts by examining the genes listed in Supplementary Table 1. Deletions (allele loss) were defined by -2 GISTIC2 thresholded score, and amplifications (allele gain) by 1 and 2 GISTIC 2 thresholded scores. Low-level hemizygous deletions (GISTIC2 score $=-1$ ) were excluded from the analysis, since single copy loss might not lead to an overt molecular phenotype for some SCNAs, due to haplo-sufficiency. The resulting data, in Table 3 , shows that genome alterations of 49 cancer-related genes are significantly associated with $F H I T$ deletion, i.e., they are lost, gained or mutated when FHIT is deleted; 24 genes were often amplified when FHIT was deleted, with KRAS and $M Y C$ being the most significant. Sixteen SCNAs were deleted in association with FHIT, with $W W O X$ as the most significant one. Nine somatically mutated genes (SMGs) were associated with FHIT loss, with TP53 most significant, and PBRMI and VHL mutations also associated with FHIT loss. Among all tested cancer genes, whether deleted, amplified or mutated, $I D H 1$ was the only one with negative $\log 2$ odds ratio, i.e. it was mutually exclusive of FHIT deletion, meaning that cancers with significantly mutated $I D H 1$ genes do not show FHIT allele loss.

\section{Signature 5 mutations are prominent in cancers with reduced $F H I T$ expression}

Alexandrov \& co-investigators have examined cancer mutational signatures in more detail in specific cancers and noted that base changes in tumor genomes could reveal the causes and paths of cancer evolution [27-29] whether due to known carcinogens or unknown processes. For example, analysis of 41 B-cell lymphoma exomes (Cell Lines Project, The Wellcome Trust Sanger Institute, COSMIC database [11] showed 67 to $>9000$ mutations comprised of mutational signatures 1, 2, $5 \& 9$. Mutational signatures $1,2 \& 9$ are caused by: aging; APOBEC family enzymes; and Ig gene hypermutation, respectively. However, the most prevalent signature in most lymphomas had an unknown cause, signature 5, which was of interest because B-cell lymphomas occurred spontaneously in Fhit ko mice [30]. Gastric, hepatocellular cancers [28], and Acute Myelogenous Leukemias (AMLs) have also shown a high frequency of signature 5 mutations, with AMLs showing nearly exclusively signature 5 [10]. These are all cancers that show large Fhit-deficient fractions [31-33]. In addition, there is evidence for a protective function of Fhit protein in gastrointestinal tumors as oral delivery of a gastrointestinal tract carcinogen, NMBA, caused a 10 -fold increase in upper gastrointestinal tumors in young Fhit ko mice [34]. 
Table 1: Correlation of SCNA genes with mutational signature 5

\begin{tabular}{|c|c|c|c|c|}
\hline Gene Symbol & $\begin{array}{c}\text { Spearman } \\
\text { Rho }\end{array}$ & Rho p & $\begin{array}{c}\text { MM } \\
\text { Regression }\end{array}$ & MM p \\
\hline \multicolumn{5}{|l|}{ Deleted genes } \\
\hline FHIT & -0.21 & $2.00 \mathrm{E}-65$ & -0.18 & $1.90 \mathrm{E}-25$ \\
\hline NAALADL2 & 0.2 & $5.40 E-63$ & 0.16 & $2.80 E-23$ \\
\hline CSMD1 & -0.1 & $2.50 \mathrm{E}-17$ & -0.08 & $1.60 \mathrm{E}-11$ \\
\hline PDE4D & -0.1 & $9.00 \mathrm{E}-17$ & -0.064 & $2.00 \mathrm{E}-05$ \\
\hline ETV6 & 0.1 & $1.10 E-15$ & 0.076 & $2.50 E-07$ \\
\hline$M A P 2 K 4$ & 0.07 & $3.20 E-09$ & 0.074 & $2.50 E-07$ \\
\hline DSCAM & -0.07 & $3.30 \mathrm{E}-09$ & -0.069 & $5.00 \mathrm{E}-06$ \\
\hline TP53 & 0.07 & $6.60 E-09$ & 0.08 & $3.80 E-08$ \\
\hline ERG & -0.07 & $1.40 \mathrm{E}-08$ & -0.064 & $1.40 \mathrm{E}-05$ \\
\hline RB1 & -0.07 & $8.10 \mathrm{E}-08$ & -0.054 & $2.40 \mathrm{E}-05$ \\
\hline RYR2 & -0.05 & $8.10 \mathrm{E}-06$ & -0.058 & $2.00 \mathrm{E}-06$ \\
\hline$M A C R O D 2$ & 0.05 & $1.90 E-05$ & 0.054 & $1.30 E-05$ \\
\hline \multicolumn{5}{|c|}{ Amplified genes } \\
\hline CCND1 & 0.08 & $1.70 \mathrm{E}-11$ & 0.092 & $3.20 \mathrm{E}-10$ \\
\hline MYCN & 0.08 & $4.20 \mathrm{E}-10$ & 0.079 & $8.80 \mathrm{E}-06$ \\
\hline CRKL & 0.07 & $2.20 \mathrm{E}-08$ & 0.063 & $2.60 \mathrm{E}-05$ \\
\hline$M D M 4$ & -0.07 & $2.60 E-08$ & -0.067 & $1.30 E-07$ \\
\hline MYC & 0.06 & $1.20 \mathrm{E}-07$ & 0.054 & $1.20 \mathrm{E}-05$ \\
\hline MCL1 & -0.06 & $1.10 E-06$ & -0.058 & $5.30 E-06$ \\
\hline
\end{tabular}

In this test, genes deleted in cancers significantly associated with mutational signature 5 were expected to show negative correlation, while for amplified genes the opposite trend was expected. Italic font notes genes with incoherent trends. Bonferroni corrected alpha of 0.05 was $2.8 \mathrm{E}-05$.

To show examples of absence of Fhit protein expression in cancers where signature 5 is the predominant mutational signature, we performed immunoblots for Fhit protein using lysates of 8 AMLs. One of the AMLs expressed some Fhit protein, likely due to $<50 \%$ blasts in the cell sample, while the others were negative or had very reduced Fhit protein expression (Figure 2A). Additionally, we examined the TCGA database for AMLs with mRNA expression data and found that FHIT mRNA expression in 153 human bone marrow-derived AML samples accessed through the Genomic Data Commons portal [35], is significantly reduced relative to 50 normal kidney tissue samples (Figure 2B).

\section{DISCUSSION}

Previous findings that led to our proposal that FHIT loss causes signature 5 mutations were: FHIT loss and signature 5 mutations occur in most types of cancer; FHIT loss and signature 5 mutations occur early in the neoplastic process; signature 5 mutations are age-associated and the expectation is that frequency of FHIT loss in a given cancer type should be age-associated due to fragile site breakage throughout life [36, 37]; loss of FHIT causes genome instability [7] and might, like loss/mutation of other caretaker genes, cause specific mutational signatures; the mutation profile of the Fhit ko mouse cells and tissues closely resembled mutational signature 5 (see Figure 3 model).

In confirmation of this proposal, we have shown that the Fhit-loss signature extracted from the exomes of ko mice has a cosine similarity of 0.89 with COSMIC signature 5. In addition, data mining of over 6,500 TCGA cancers identified FHIT as the gene exhibiting the most significant, and negative, correlation with mutational signature 5 (Spearman $\mathrm{p}=2.00 \mathrm{E}-65$, MM Regression $\mathrm{p}=$ 1.90E-25). SCNAs in other fragile genes do not correlate with Signature 5. Confirming this specificity, we found 
Table 2: Correlation of FHIT loss with mutational signatures in TCGA cohorts

\begin{tabular}{|c|c|c|c|c|c|}
\hline Signature & $\begin{array}{c}\text { FHIT loss \& } \\
\text { substitution rate } \\
\text { Spearman Rho }\end{array}$ & Rho p & $\mathbf{N}$ & MM regression & MM p \\
\hline Sign. 5 & -0.21 & $2.00 \mathrm{E}-65$ & 6649 & -0.18 & $1.9 \mathrm{E}-25$ \\
\hline Sign. 2 & -0.22 & $5.30 \mathrm{E}-43$ & 3702 & -0.18 & $1.1 \mathrm{E}-21$ \\
\hline Sign.7 & 0.52 & $4.60 \mathrm{E}-68$ & 975 & ns & ns \\
\hline Sign. 13 & -0.11 & $7.20 \mathrm{E}-08$ & 2234 & ns & ns \\
\hline Sign.3 & -0.10 & $1.20 \mathrm{E}-05$ & 1757 & ns & ns \\
\hline Sign.6 & 0.05 & 0.001 & 3629 & ns & ns \\
\hline Sign.26 & 0.08 & 0.002 & 1546 & $\mathrm{~ns}$ & ns \\
\hline Sign. 17 & -0.08 & 0.003 & 1301 & ns & ns \\
\hline Sign. 18 & -0.15 & 0.006 & 337 & ns & ns \\
\hline Sign. 8 & -0.06 & 0.045 & 974 & ns & ns \\
\hline
\end{tabular}

The table shows the significant correlation values of FHIT allele losses (only homozygous deletions were used) with 10 different COSMIC mutational signatures. All TCGA cancer cohorts with available data were analyzed. $\mathrm{N}$ is total number of cancer samples exhibiting the specific signature (see Supplementary Table 3 for cancers exhibiting the specific signatures); ns, not significant.

that Fhit loss correlated only with Signature 5 and Signature 2, the A3B signature, as expected given that Fhit loss generates ssDNA substrates for A3B hypermutation [9]. Lastly, we identify other cancer-associated genes that negatively or positively correlate with Fhit loss.

A telling study, also in accord with the strong association of FHIT loss with mutational signature 5, was the recent report of the mutational consequences of smoking [39], comparing somatic mutations in smokers $v s$ nonsmokers for lung and other cancers known to be increased in cigarette smokers (Figure 4, examples from Figure 2 of ref 39). Increases in smokers $v s$ nonsmokers were reported for signatures $2 \& 13$ (APOBEC), signature 4 (C>A mutations due to tobacco smoke carcinogens), $5 \& 16$ (origins unknown). These mutation increases were apparently of clonal mutations for signatures 4 and 5 [39], due to smoke exposure during preneoplastic stages. In Figure 4A, mutational signature 5 showed mutations across all 96 mutation subtypes, with more $\mathrm{T}>\mathrm{C}$ and $\mathrm{C}>\mathrm{T}$ mutations, similar to the signature of Fhit ko tissues (Figure 1). Signature 5 mutations were found in all cancer types studied in this report [39], including the cancers of the nonsmokers, as expected for cancers with FHIT loss. Signature 5 mutations were increased in smokers $v s$ nonsmokers in lung, larynx, pharynx, oral cavity, esophageal squamous, bladder, liver, cervical and kidney cancers [39] in a non-age related manner, since carcinogens in cigarette smoke would have compounded the signature 5/FHIT loss signature, as noted in the Figure
3 model. These are all cancers for which reduced Fhit expression has been reported [2, 40-45]. In considering Figure 4, there are striking findings consistent with a role for FHIT loss in production of signature 5 mutations. First, these alterations can occur early in the preneoplastic process and would appear as clonal alterations in a tumor, just as alterations within the fragile FHIT locus are clonal in cancers and cancer cell lines [1, 46, 47]. FHIT alterations and loss of expression occur more frequently in precancerous lung tissues of smokers [2, 40, 41, 48], such that lung adenocarcinomas would show FHIT loss unrelated to age at diagnosis. We also know from the study of Waters et al [9], that loss of FHIT expression creates optimal ssDNA substrates for A3B enzyme activity, and in many smoker and nonsmoker cancers of the cervix, mutational signature 5 occurs in the same tumors as APOBEC signature $2 \& 13$ mutations (Figure 4A panels). This is also true in the oral cavity and bladder cancers of smokers [39], all cancers for which Fhit protein expression is lost or reduced in large fractions of cases. The kidney cancer results (ref 39 \& Figure 4A) where the mutation burdens are low in smoking associated and non-associated cancers, with signature 5 as the main signature observed (Figure 4), is satisfying at the genetic level since the FHIT gene was originally cloned from cells of an individual at high risk for multifocal, bilateral clear cell kidney cancer that occurred in family members carrying an inherited, balanced chromosome translocation between chromosomes $3 \& 8$, where the chromosome 3 
Table 3: Genes altered in association with $F H I T$ allele loss

\begin{tabular}{|c|c|c|}
\hline Gene Symbol & Log2 Odds Ratio & Fisher test $p$ \\
\hline \multicolumn{3}{|c|}{ Somatically mutated genes ${ }^{a}$} \\
\hline TP53 & 1.51 & $4.00 \mathrm{E}-12$ \\
\hline PBRM1 & 2.25 & $1.50 \mathrm{E}-06$ \\
\hline MLL2 & 1.46 & $5.50 \mathrm{E}-06$ \\
\hline VHL & 2.16 & $5.90 \mathrm{E}-06$ \\
\hline MLL4 & 1.56 & $6.60 \mathrm{E}-05$ \\
\hline SMC3 & 2.3 & 7.10E-05 \\
\hline IDH1 & -2.16 & 7.10E-05 \\
\hline CREBBP & 1.46 & 0.00012 \\
\hline LRRK2 & 1.45 & 0.00017 \\
\hline \multicolumn{3}{|c|}{ Amplified genes $^{b}$} \\
\hline KRAS & 1.8 & $4.50 \mathrm{E}-14$ \\
\hline MYC & 1.59 & $3.90 \mathrm{E}-13$ \\
\hline HMGA2 & 1.75 & $2.10 \mathrm{E}-12$ \\
\hline MYCN & 1.86 & $8.20 \mathrm{E}-12$ \\
\hline NCOA3 & 1.47 & $1.00 \mathrm{E}-11$ \\
\hline BCL2L1 & 1.47 & $1.00 \mathrm{E}-11$ \\
\hline TERT & 1.61 & $1.90 \mathrm{E}-11$ \\
\hline MDM2 & 1.64 & $3.00 \mathrm{E}-11$ \\
\hline IGF1R & 1.94 & $5.10 \mathrm{E}-11$ \\
\hline CDK4 & 1.57 & $1.60 \mathrm{E}-10$ \\
\hline CCNE1 & 1.52 & $1.80 \mathrm{E}-09$ \\
\hline FGFR1 & 1.5 & $2.60 \mathrm{E}-09$ \\
\hline CCND1 & 1.42 & $1.70 \mathrm{E}-08$ \\
\hline SKP2 & 1.28 & $2.00 \mathrm{E}-07$ \\
\hline MYB & 1.63 & $4.50 \mathrm{E}-06$ \\
\hline JUN & 1.45 & $1.30 \mathrm{E}-05$ \\
\hline CDK6 & 0.94 & $1.70 \mathrm{E}-05$ \\
\hline EGFR & 0.9 & $3.30 \mathrm{E}-05$ \\
\hline AR & 1.19 & $3.90 \mathrm{E}-05$ \\
\hline LMO1 & 1.37 & $7.00 \mathrm{E}-05$ \\
\hline PDGFRA & 1.26 & 0.00035 \\
\hline KIT & 1.3 & 0.00036 \\
\hline BIRC2 & 1.11 & 0.00043 \\
\hline YAP1 & 1.1 & 0.00044 \\
\hline \multicolumn{3}{|l|}{ Deleted genes $^{c}$} \\
\hline WWOX & 3.66 & $1.30 \mathrm{E}-30$ \\
\hline PDE4D & 3.67 & $6.00 \mathrm{E}-25$ \\
\hline PTPRD & 3.27 & $1.60 \mathrm{E}-21$ \\
\hline
\end{tabular}

(Continued) 


\begin{tabular}{lcc}
\hline Gene Symbol & Log2 Odds Ratio & Fisher test p \\
\hline NAALADL2 & 4.62 & $5.70 \mathrm{E}-19$ \\
MACROD2 & 3.17 & $1.30 \mathrm{E}-12$ \\
LRP1B & 2.96 & $4.00 \mathrm{E}-12$ \\
PTPRN2 & 4.11 & $1.50 \mathrm{E}-10$ \\
PARK2 & 2.44 & $7.80 \mathrm{E}-08$ \\
CSMD1 & 1.9 & $8.70 \mathrm{E}-07$ \\
ATM & 2.92 & $2.50 \mathrm{E}-06$ \\
DMD & 2.37 & $2.60 \mathrm{E}-06$ \\
CDKN2A & 1.35 & $5.10 \mathrm{E}-06$ \\
PRKG1 & 2.61 & $3.20 \mathrm{E}-05$ \\
CNTNAP2 & 2.8 & $3.40 \mathrm{E}-05$ \\
DLG2 & 2.69 & 0.00035 \\
PARD3B & 2.4 & 0.00041 \\
\hline
\end{tabular}

$\log 2$ OR $>0$ means that the cancer event was more frequent in tumors with FHIT deletion than in those with normal FHIT. Log2 OR $<0$ means the event was mutually exclusive of FHIT deletion. Fisher exact test was performed to evaluate association of cancer events. ${ }^{a}$ Mutated gene score $=1$, wt score $=0$. Bonferroni correction for 189 tests (alpha $\left.=0.05\right), p$ threshold $=0.0003 .{ }^{b}$ Bonferroni correction for 33 tests (alpha $=0.05$ ), $\mathrm{p}$ threshold $=0.001$. ${ }^{\mathrm{c}}$ Samples that had low-level deletions (GISTIC -1) were not included in this analysis. Bonferroni correction for 45 tests $($ alpha $=0.05), p$ threshold $=$ 0.001 .
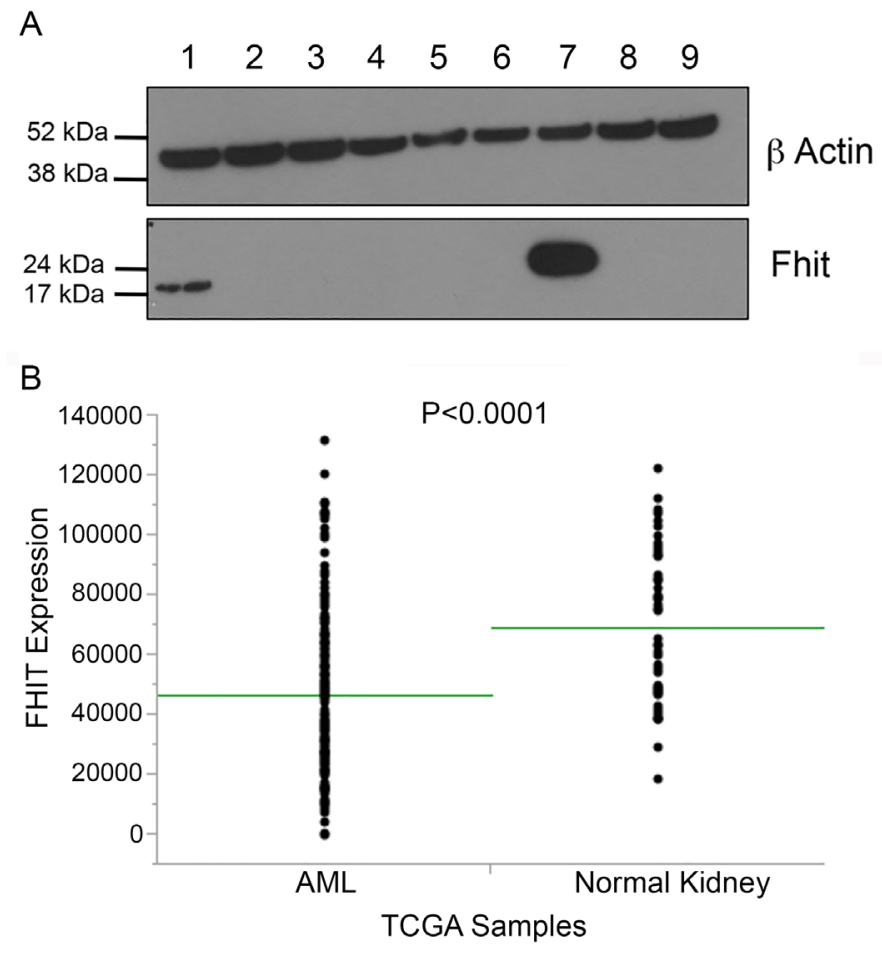

Figure 2: Fhit expression in Acute Myelogenous Leukemias, a cancer exhibiting only mutational signatures 1 and 5. (A) Immunoblot depicting loss of Fhit protein expression in 7 of 8 AML bone marrow samples (lanes 1-6, 8 \& 9), positive control H1299 D1 cells with induced Fhit expression (lane 7). (B) Fhit expression in samples accessed through Genome Data Commons, depicting reduced FHIT RNA expression in 153 AMLs derived from bone marrow vs 50 kidney samples derived from normal tissue. Neither normal bone marrow nor matched peripheral white blood cell RNA was available for comparison. 
translocation break was within the fragile FRA3B locus, interrupting the FHIT gene [1].

As for the originating mechanism for the Fhit loss signature, and theoretically for signature 5 , it may arise partly because of the down-regulation of TK1 expression by loss of Fhit expression and subsequent genome instability [7]. The signatures compiled from exome sequences of Fhit ko kidney and lung tissue and ko kidney cell lines revealed accumulation of $>2000$ SBSs per exome sequence and increased numbers of $\mathrm{C}>\mathrm{T}$ and $\mathrm{T}>\mathrm{C}$ SBSs, with a signature highly similar to mutational signature 5. As noted previously [21], C $>\mathrm{T}$ transitions may be generated through spontaneous deamination or DNA replication errors at $\mathrm{CpG}$ dinucleotides. SBS peaks for ACG, CCG, GCG, \& TCG sequences, where the central $\mathrm{C}$ transitions to $\mathrm{T}$, include such dinucleotide sequences. The $\mathrm{T}>\mathrm{C}$ transitions observed in Fhit ko tissues may be due to the increased ratio of dUTP:TTP, allowing misincorporation of dUTP in place of TTP. Depending on the involved translesion polymerase, DNA replication might insert a guanine or a cytosine across from abasic sites and after another round of DNA replication, will result in a $\mathrm{T}>\mathrm{C}$ or a $\mathrm{T}>\mathrm{G}$ SBS $[7,20,49-51]$.

\section{Clues to Fhit loss/signature 5 association}

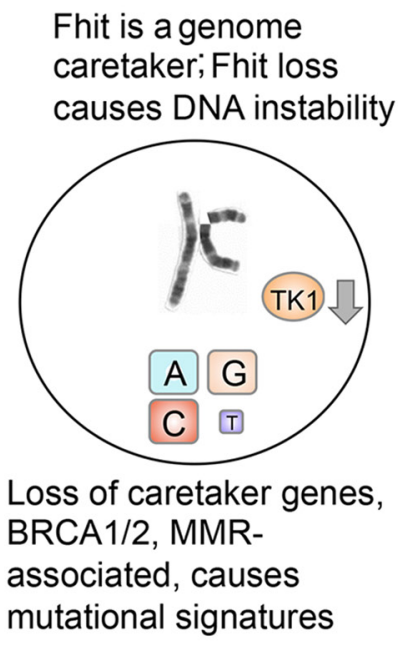

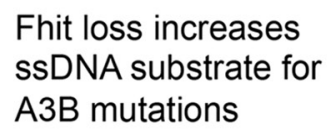

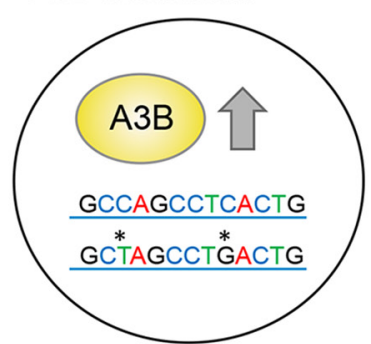

A3B signature 2 occurs with signature 5 in most cancers

\section{Fhit ko exome sequences show $\mathrm{C}>\mathrm{T}$ and $\mathrm{T}>\mathrm{C}$ SBSs}

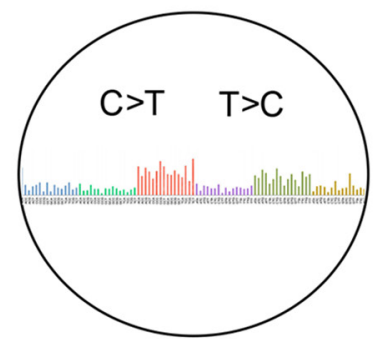

Fhit loss mutation profile resembles signature 5

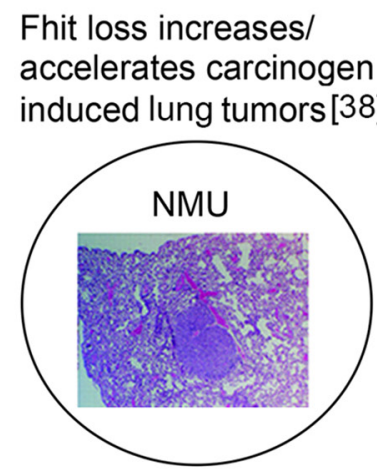

Signature 5 mutations greatly increased in cancers of smokers

Figure 3: Features of cells with FHIT loss (above circles), and specific mutational signatures (below circles).

A
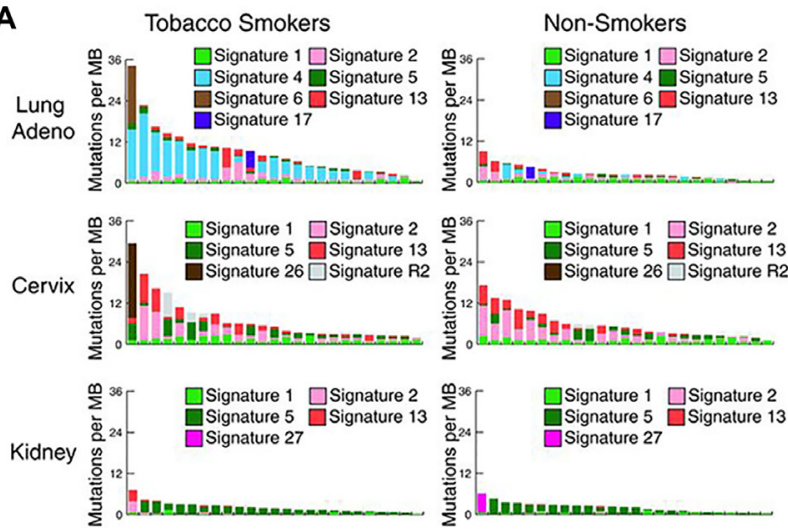

B
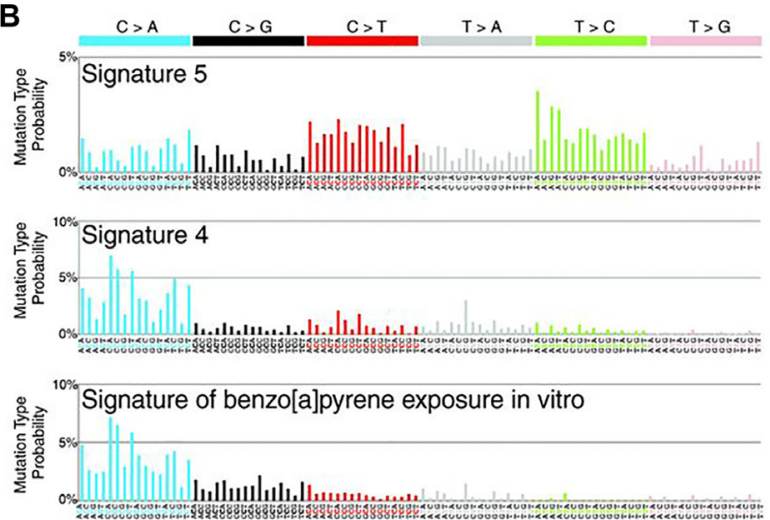

Figure 4: The mutational signatures in smokers vs nonsmokers. An abbreviated copy of the "smoking signatures" from Figure 2 of ref 39 ffrom Alexandrov LB et al. 2016. Mutational signatures associated with tobacco smoking in human cancer. Science 354: 618622. Reprinted with permission from AAAS \}, to emphasize features of this signature that make FHIT loss a strong candidate as cause of mutational signature 5: (A) illustration of the mutation spectra in 25 randomly selected cancer genomes (individual bars from smokers or nonsmokers of a given cancer type). Each bar is colored proportionately to the number of mutations/Mb of the specific mutational signatures found in the sample genome. (B) The pattern of mutational signatures observed in tobacco smoker cancers. 
Kim et al [21] have previously examined the mutational processes in urothelial cancer, a cancer in which the NER gene ERCC2 is significantly mutated, and proposed that such tumor cohorts demonstrate a strong association between somatic ERCC2 mutations and mutational signature 5 . These investigators noted an association of signature 5 mutations and smoking that was not associated with ERCC2 mutation status, while there is strong evidence that FHIT loss is associated with exposure to cigarette smoke $[2,40,48]$. Since Fhit is a genome caretaker protein, it is possible that its loss, which occurs frequently in bladder cancer [45], could predispose to the ERCC2 mutations associated with these cancers.

Tomasetti \& Vogelstein $[52,53]$ have recently reported that 'Variation in cancer risk among tissues can be explained by the number of stem cell divisions', and that these mutations are responsible for $2 / 3$ of the mutations in human cancers. The authors emphasize the importance of early detection and intervention to reduce disease and death for the cancers arising from 'unavoidable' mutations that are a result of errors during replication stress. We have presented data here that supports the hypothesis that FHIT loss is the underlying determinant of mutational signature 5 , a signature that is ubiquitous in human cancers. We propose that many of the 'unavoidable' mutations in cancer, such as those significantly associated with FHIT loss in Table 3, are due to the genome instability introduced through FHIT allele alterations caused by replicative stress at the FRA3B locus. If replicative stress at this fragile site or the results of such stress could be prevented, many 'unavoidable' mutations might be avoided.

Most cancer research is currently focused on finding targets for curing patients with progressive or advanced cancer; thus these studies are looking for cancer driver genes, which may be targeted by specific drugs. At the same time, investigators should be considering prevention of new cancers. As noted above, Tomassetti \& Vogelstein $[52,53]$ have proposed that $2 / 3$ of mutations in cancer may be unavoidable, a result of errors during replication. Many of these 'unavoidable' mutations, signature 5 mutations, likely associated with replication stress occurring during and after FHIT allele alteration, may in fact be avoidable if we can target the genome instability associated with FHIT loss, for example by low dose thymidine supplementation. At the very least, onset of preneoplasia in at-risk tissues might be predicted by following FHIT gene alterations by RT-qPCR [1]. With very early detection, such cancers would be more easily cured.

\section{MATERIALS AND METHODS}

\section{Data mining, computational methods and statistics}

Publicly available mutational signatures were obtained for all TCGA samples examined for mutational signatures, using the Wellcome Trust Sanger Institute algorithm by Alexandrov et al [12]. The molecular portraits of TCGA samples, including somatic mutations and copy number alterations (SCNA as scored by GISTIC2; Genomic Identification of Significant Targets in Cancer, version 2.0) were obtained from Firebrowse (http://firebrowse.org). The list of the frequent SCNAs in cancer were obtained from the SCNA list of Beroukhim et al [23] and completed with the deleted and amplified cancer driver genes described by Vogelstein et al [24]. See Supplementary Table 1 for listing of the 87 total genes, including FHIT, 44 deleted, 31 amplified and 12 mutated.

The thresholded GISTIC2 scores for the SCNA cancer genes obtained from FireBrowse were investigated for association with mutational signatures, using Spearman correlation and MM regression. Highly stringent Bonferroni correction was implemented, dividing the critical P value $(\alpha=0.05)$ by the number of comparisons being made (i.e. 87 SCNA genes and 24 signatures).

Spearman correlation and MM robust regression (Robustbase package in R; this function computes an MM-type regression estimator as described [25, 26] were computed for lists generated in Tables 1, 2 and 3. We chose DNA copy number variation as the most robust method to establish a link between FHIT and cancer signatures. FHIT does not exhibit somatic mutations and Fhit protein expression data are not available in large-scale cancer studies, such as in TCGA.

\section{Tissues, cell lines and exome sequences}

The Fhit+/+ and -/- tissues and cell lines from the constitutive ko strain have been described, as have the exome sequence results and analyses [20]. Females of the constitutive Fhit $^{-/}$strain and our laboratory B6 strain, were superovulated by hormone treatment, mated and embryos collected for isolation of inner cell mass cells and establishment, characterization and freezing of embryonic stem cell lines. The ESC cells were grown briefly in stem cell culture conditions before isolating DNA for exome sequencing; some ESC cells were then switched to non stem cell medium and allowed to differentiate through several subcultures before preparing DNA for sequencing. The exome sequencing data for the ESCs has been added to SRA BioProject PRJNA260539 (will be released on or before publication of this manuscript), where sequence files for the other Fhit ko samples are already available.

Filtered SBS numbers in wt DNAs ranged between 19 and $\sim 300$, numbers too low to reliably examine in the SomaticSignatures algorithm [22]. Whole exome sequences of the following tissues and cell lines exhibited $>2500$ SBSs each and were analyzed for mutational signatures as described $[10,11,22]$ : ko kidney tissue, 2912 SBSs; ko lung tissue, 2596; NS1 ko kidney cell line, 4631; NS4 ko kidney cell line, 4757; ko ESC line, 3521; ko ESC derived differentiated line, 3028. Mutational 
signatures were derived from these six samples using the SomaticSignatures $\mathrm{R}$ package [22] and $\mathrm{R}$ version 3.2.4 for Windows. Mutation spectra were decomposed using NMF [54]; signature decomposition was determined for 2, 3, or 4 signatures. Manual examination of signatures, and comparison with previously published signatures $[12,54]$, was used to determine that two was the number of signatures readily noted in the Fhit ko exome sequences. The SomaticSignatures package [22] was used to generate mutational signatures from the six Fhit ko samples described above. The matrix and NMF_fitted_Spectrum generated by SomaticSignatures is shown in Supplementary Table 4 and Supplementary Figure 1, respectively. Information for the use of SomaticSignatures was found at https://bioconductor.org/ packages/release/bioc/vignettes/SomaticSignatures/inst/ doc/SomaticSignatures-vignette.html.

\section{Fhit expression analyses in cancers}

Immunoblotting was performed as described using anti-beta actin (sc-1616) and polyclonal rabbit antiFhit serum [7]. The deidentified human AML samples were obtained from the OSUCCC leukemia tissue bank. For FHIT RNA expression analysis, expression files for 153 bone marrow-derived AML samples and 50 normal kidney samples were chosen at random and the Fragments Per Kilobase of transcript per million mapped reads files, normalized to upper quartile (FPKM-UQ), were downloaded from Genome Data Commons [35]. The expression values were plotted in JMP and a nonparametric Wilcoxon two-sample test was performed (depicted in Figure 2B).

\section{Abbreviations}

A3B: APOBEC3B; COSMIC: Catalog of Somatic Mutations in Cancer; SBS: single base substitution; CNAs:copy number alterations; wt: wild-type; ko: knockout; NMF: non-negative matrix factorization; ESC: embryonic stem cell; SCNAs: Somatic Copy Number Alterations; TCGA: The Cancer Genome Atlas; SMGs: somatically mutated genes; AMLs: Acute Myelogenous Leukemias.

\section{Author contributions}

SV designed \& executed the association studies for FHIT allele loss \& mutational signatures, performed the associated statistical analyses \& prepared the Tables. TD \& CAP performed the mutational signature analysis of the mouse exome sequences, \& associated 'cosine' calculations; MSS performed FHIT expression analyses in AMLs; KH conceived the hypothesis, worked with co-authors to choose methods of analysis \& wrote the manuscript, with SV, TD \& MSS. All authors contributed specific text sections \& editing of the manuscript; TD \& MSS prepared the figures.

\section{ACKNOWLEDGMENTS}

Many thanks to Ludmil B. Alexandrov for kindly reading an early draft $\mathrm{MS}$. We also thank former lab members, Joshua C. Saldivar, Catherine E. Waters, Satoshi Miuma \& Jenna R. Karras, whose research results were the building blocks for the hypothesis that FHIT loss underlies signature 5 mutations; Ramiro Garzon \& laboratory members kindly contributed the AML samples. We gratefully acknowledge the contributions of Drs. Sigrid Eckardt \& K. John McLaughlin of Nationwide Children's Hospital, Columbus $\mathrm{OH}$, in isolating the mouse strain ESC cell lines.

\section{CONFLICTS OF INTEREST}

The authors confirm that there are no conflicts of interest.

\section{FUNDING}

This work was supported by an OSUCCC Pelotonia Graduate fellowship (to MSS), CA154200 (KH), CA120516 (KH) from the National Institutes of Health of the US Public Health Service, Pelotonia Idea grants (to $\mathrm{KH}$ ), and the National Institutes of Health, National Library of Medicine Biomedical and Health Informatics Training Program at the University of Washington (CAP, Grant Nr. T15LM007442).

\section{REFERENCES}

1. Ohta M, Inoue H, Cotticelli MG, Kastury K, Baffa R, Palazzo J, Siprashvili Z, Mori M, McCue P, Druck T, Croce $\mathrm{CM}$, Huebner K. The FHIT gene, spanning the chromosome 3 p14.2 fragile site and renal carcinoma-associated $t(3 ; 8)$ breakpoint, is abnormal in digestive tract cancers. Cell. 1996; 84:87-97.

2. Sozzi G, Pastorino U, Moiraghi L, Tagliabue E, Pezzella F, Ghirelli C, Tornielli S, Sard L, Huebner K, Pierotti MA, Croce CM, Pilotti S. Loss of FHIT function in lung cancer and preinvasive bronchial lesions. Cancer Res. 1997; 58:5032-7.

3. Gorgoulis VG, Vassiliou LV, Karakaidos P, Zacharatos P, Kotsinas A, Liloglou T, Venere M, Ditullio RA Jr, Kastrinakis NG, Levy B, Kletsas D, Yoneta A, Herlyn M, et al. Activation of the DNA damage checkpoint and genomic instability in human precancerous lesions. Nature. 2005; 434:907-13.

4. Guler G, Uner A, Guler N, Han SY, Iliopoulos D, McCue $\mathrm{P}$, Huebner K. Concordant loss of fragile gene expression 
early in breast cancer development. Pathol Int. 2005; 55:471-8.

5. Bartkova J, Horejsí Z, Koed K, Krämer A, Tort F, Zieger K, Guldberg P, Sehested M, Nesland JM, Lukas C, Ørntoft T, Lukas J, Bartek J. DNA damage response as a candidate anti-cancer barrier in early human tumorigenesis. Nature 2005; 434:864-70.

6. Fassan M, Rusev B, Corbo V, Gasparini P, Luchini C, Vicentini C, Mafficini A, Paiella S, Salvia R, Cataldo I, Scarpa A, Huebner K. Fhit down-regulation is an early event in pancreatic carcinogenesis. Virchows Arch. 2017; 470:647-653.

7. Saldivar JC, Miuma S, Bene J, Hosseini SA, Shibata H, Sun J, Wheeler LJ, Mathews CK, Huebner K. Initiation of genome instability and preneoplastic processes through loss of Fhit expression. PLoS Genet. 2012; 8:e1003077.

8. Saldivar JC, Bene J, Hosseini SA, Miuma S, Horton S, Heerema NA, Huebner K. Characterization of the role of Fhit in suppression of DNA damage. Adv Biol Regul. 2013; 53:77-85.

9. Waters CE, Saldivar JC, Amin ZA, Schrock MS, Huebner K. FHIT loss-induced DNA damage creates optimal APOBEC substrates: Insights into APOBEC-mediated mutagenesis. Oncotarget. 2015; 6:3409-19. http://doi. org/10.18632/oncotarget.2636.

10. Alexandrov LB, Nik-Zainal S, Wedge DC, Aparicio SA, Behjati S, Biankin AV, Bignell GR, Bolli N, Borg A, Børresen-Dale AL, Boyault S, Burkhardt B, Butler AP, et al. Signatures of mutational processes in human cancer. Nature. 2013; 500:415-21.

11. Forbes SA, Beare D, Boutselakis H, Bamford S, Bindal N, Tate J, Cole CG, Ward S, Dawson E, Ponting L, Stefancsik R, Harsha B, Kok CY, et al. COSMIC: somatic cancer genetics at high-resolution. Nucleic Acids Res. 2017; 45:D777-D783.

12. Alexandrov LB, Jones PH, Wedge DC, Sale JE, Campbell PJ, Nik-Zainal S, Stratton MR. Clock-like mutational processes in human somatic cells. Nat Genet. 2015; 47:1402-7

13. Rahbari R, Wuster A, Lindsay SJ, Hardwick RJ, Alexandrov LB, Al Turki S, Dominiczak A, Morris A, Porteous D, Smith B, Stratton MR. Timing, rates and spectra of human germline mutation. Nat Genet. 2016; 48:126-33.

14. Stratton MR, Campbell PJ, Futreal PA. The cancer genome. Nature. 2009;458:719-24.

15. Palumbo E, Tosoni E, Matricardi L, Russo A. Genetic instability of the tumor suppressor gene FHIT in normal human cells. Genes Chrom Cancer. 2013; 52:832-44.

16. Hosseini SA, Horton S, Saldivar JC, Miuma S, Stampfer MR, Heerema NA, Huebner K. Common chromosome fragile sites in human and murine epithelial cells and FHIT/ FRA3B loss-induced global genome instability. Genes Chrom Cancer. 2013; 52:1017-29.

17. Le Tallec B, Millot GA, Blin ME, Brison O, Dutrillaux B, Debatisse M. Common fragile site profiling in epithelial and erythroid cells reveals that most recurrent cancer deletions lie in fragile sites hosting large genes. Cell Rep. 2013; $4: 420-8$.

18. Miuma S, Saldivar JC, Karras JR, Waters CE, Paisie CA, Wang Y, Jin V, Sun J, Druck T, Zhang J, Huebner K. Fhit deficiency-induced global genome instability promotes mutation and clonal expansion. PLoS One. 2013; 8:e80730.

19. Karras JR, Schrock MS, Batar B, Zhang J, La Perle K, Druck T, Huebner K. Fhit loss-associated initiation and progression of neoplasia in vitro. Cancer Sci. 2016; 107:1590-8.

20. Paisie CA, Schrock MS, Karras JR, Zhang J, Miuma S, Ouda IM, Waters CE, Saldivar JC, Druck T, Huebner K. Exome-wide single-base substitutions in tissues and derived cell lines of the constitutive Fhit knockout mouse. Cancer Sci. 2016; 107:528-35.

21. Kim J, Mouw KW, Polak P, Braunstein LZ, Kamburov A, Tiao G, Kwiatkowski DJ, Rosenberg JE, Van Allen EM, D'Andrea AD, Getz G. Somatic ERCC2 mutations are associated with a distinct genomic signature in urothelial tumors. Nat Genet. 2016; 48:600-6.

22. Gehring JS, Fischer B, Lawrence M, Huber W. SomaticSignatures: Inferring mutational signatures from single-nucleotide variants. Bioinformatics. 2015; 31:3673-5.

23. Beroukhim R, Mermel CH, Porter D, Wei G, Raychaudhuri S, Donovan J, Barretina J, Boehm JS, Dobson J, Urashima M, Mc Henry KT, Pinchback RM, Ligon AH, et al. The landscape of somatic copy-number alteration across human cancers. Nature. 2010; 463:899-905.

24. Vogelstein B, Papadopoulos N, Velculescu VE, Zhou S, Diaz LA Jr, Kinzler KW. Cancer genome landscapes. Science. 2013; 339:1546-58. Review.

25. Koller M, Stahel WA. Sharpening Wald-type inference in robust regression for small samples, Computational Statistics \& Data Analysis. 2011; 55:2504-15.

26. Yohai VJ. High breakdown-point and high efficiency estimates for regression. The Annals of Statistics. 1987; 15:642-65.

27. Schulze K, Imbeaud S, Letouzé E, Alexandrov LB, Calderaro J, Rebouissou S, Couchy G, Meiller C, Shinde J, Soysouvanh F, Calatayud AL, Pinyol R, Pelletier L, et al. Exome sequencing of hepatocellular carcinomas identifies new mutational signatures and potential therapeutic targets. Nat Genet. 2015; 47:505-11.

28. Wagener R, Alexandrov LB, Montesinos-Rongen M, Schlesner M, Haake A, Drexler HG, Richter J, Bignell GR, McDermott U, Siebert R. Analysis of mutational signatures in exomes from B-cell lymphoma cell lines suggest APOBEC 3 family members to be involved in the pathogenesis of primary effusion lymphoma. Leukemia. 2015; 29:1612-5.

29. Alexandrov LB, Nik-Zainal S, Siu HC, Leung SY, Stratton MR. A mutational signature in gastric cancer suggests therapeutic strategies. Nat Commun. 2015; 6:8683. 
30. Zanesi N, Fidanza V, Fong LY, Mancini R, Druck T, Valtieri M, Rüdiger T, McCue PA, Croce CM, Huebner K. The tumor spectrum in FHIT-deficient mice. Proc Natl Acad Sci U S A. 2002; 98:10250-9.

31. Capuzzi D, Santoro E, Hauck WW, Kovatich AJ, Rosato FE, Baffa R, Huebner K, McCue PA. Fhit expression in gastric adenocarcinoma: correlation with disease stage and survival. Cancer. 2000; 88:24-34.

32. Zhang X, Li HM, Liu Z, Zhou G, Zhang Q, Zhang T, Zhang J, Zhang C. Loss of heterozygosity and methylation of multiple tumor suppressor genes on chromosome 3 in hepatocellular carcinoma. J Gastroenterol. 2013; 48:132-43.

33. Paulsson K, An Q, Moorman AV, Parker H, Molloy G, Davies T, Griffiths M, Ross FM, Irving J, Harrison CJ, Young BD, Strefford JC. Methylation of tumour suppressor gene promoters in the presence and absence of transcriptional silencing in high hyperdiploid acute lymphoblastic leukaemia. J Haematol. 2009; 144:838-47.

34. Fong LY, Fidanza V, Zanesi N, Lock LF, Siracusa LD, Mancini R, Siprashvili Z, Ottey M, Martin SE, Druck T, McCue PA, Croce CM, Huebner K. Muir-Torre-like syndrome in Fhit-deficient mice. Proc Natl Acad Sci U S A. 2000; 97:4742-7.

35. Grossman RL, Heath AP, Ferretti V, Varmus HE, Lowy DR, Kibbe WA, Staudt LM. Toward a shared vision for cancer genomic data. New England J Med. 2016; 375:1109-1112.

36. Ardisia C, Venti G, Colozza MA, Breschi C, Porfirio B, Davis S, Tonato M, Donti E. Expression of aphidicolininduced fragile sites in lymphocytes of patients with breast cancer. Cancer Genet Cytogenet. 1993; 67:113-6.

37. Furuya $\mathrm{T}$, Ochi H, Watanabe S. Common fragile sites in chromosomes of bone marrow cells and peripheral blood lymphocytes from healthy persons and leukemia patients. Cancer Genet Cytogenet. 1989; 43:131-8.

38. Zanesi N, Mancini R, Sevignani C, Vecchione A, Kaou M, Valtieri M, Calin GA, Pekarsky Y, Gnarra JR, Croce CM, Huebner K. Lung cancer susceptibility in Fhit-deficient mice is increased by Vhl haploinsufficiency. Cancer Res. 2005; 65:6576-82.

39. Alexandrov LB, Ju YS, Haase K, Van Loo P, Martincorena I, Nik-Zainal S, Totoki Y, Fujimoto A, Nakagawa H, Shibata T, Campbell PJ, Vineis P, Phillips DH, Stratton MR. Mutational signatures associated with tobacco smoking in human cancer. Science. 2016; 354:618-22.

40. Sozzi G, Sard L, De Gregorio L, Marchetti A, Musso K, Buttitta F, Tornielli S, Pellegrini S, Veronese ML, Manenti G, Incarbone M, Chella A, Angeletti CA, et al. Association between cigarette smoking and FHIT gene alterations in lung cancer. Cancer Res. 1998; 57:2121-3.

41. Mao L, Lee JS, Kurie JM, Fan YH, Lippman SM, Lee JJ, Ro JY, Broxson A, Yu R, Morice RC, Kemp BL, Khuri $\mathrm{FR}$, Walsh GL, et al. Clonal genetic alterations in the lungs of current and former smokers. J Natl Cancer Inst. 1997; 89:857-62.
42. Otero-Garcia JE, Youssef E, Enamorado II, Du W, Yoo GH, Merati K, Kewson D, Lonardo F, Jacobs JR, Kim H. Prognostic significance of p53 and FHIT in advanced oropharyngeal carcinoma. Am J Otolaryngol. 2004; 25:231-9.

43. Heerden WF, Swart TJ, van Heerden MB, van Rensburg EJ, Engelbrecht S, Dreyer L, Huebner K. Immunohistochemical evaluation of Fhit protein expression in oral squamous cell carcinomas. J Oral Pathol Med. 1999; 28:433-7.

44. Miyawaki Y, Kawachi H, Ooi A, Eishi Y, Kawano T, Inazawa $\mathrm{J}$, Imoto I. Genomic copy-number alterations of MYC and FHIT genes are associated with survival in esophageal squamous-cell carcinoma. Cancer Sci. 2012; 103:1558-66.

45. Baffa R, Gomella LG, Vecchione A, Bassi P, Mimori K, Sedor J, Calviello CM, Gardiman M, Minimo C, Strup SE, McCue PA, Kovatich AJ, Pagano F, et al. Loss of FHIT expression in transitional cell carcinoma of the urinary bladder. Am J Pathol. 2000; 156:419-24.

46. Mimori K, Druck T, Inoue H, Alder H, Berk L, Mori M, Huebner K, Croce CM. Cancer-specific chromosome alterations in the constitutive fragile region FRA3B. Proc Natl Acad Sci U S A. 1999; 96:7456-7461. Erratum in: Proc Natl Acad Sci U S A. 1999; 96:10944.

47. Matsuyama A, Shiraishi T, Trapasso F, Kuroki T, Alder H, Mori M, Huebner K, Croce CM. Fragile site orthologs FHIT/FRA3B and Fhit/Fra14A2: evolutionarily conserved but highly recombinogenic. Proc Natl Acad Sci U S A. 2003; 100:14988-93.

48. Nelson HH, Wiencke JK, Gunn L, Wain JC, Christiani DC, Kelsey KT. Chromosome 3p14 alterations in lung cancer: evidence that FHIT exon deletion is a target of tobacco carcinogens and asbestos. Cancer Res. 1998; 58:1804-7.

49. Guillet M, Boiteux S. Origin of endogenous DNA abasic sites in Saccharomyces cerevisiae. Mol Cell Biol. 2003; 23:8386-94.

50. Waters LS, Minesinger BK, Wiltrout ME, D'Souza $\mathrm{S}$, Woodruff RV, Walker GC. Eukaryotic translesion polymerases and their roles and regulation in DNA damage tolerance. Microbiol Mol Biol Rev. 2009; 73:134-54.

51. Prakash S, Johnson RE, Prakash L. Eukaryotic translesion synthesis DNA polymerases: specificity of structure and function. Annu Rev Biochem. 2005; 74:317-53.

52. Tomasetti C, Vogelstein B. Cancer etiology. Variation in cancer risk among tissues can be explained by the number of stem cell divisions. Science. 2015; 347:78-81.

53. Tomasetti C, Li L, Vogelstein B. Stem cell divisions, somatic mutations, cancer etiology, and cancer prevention. Science. 2017; 355:1330-4.

54. Nik-Zainal S, Davies H, Staaf J, Ramakrishna M, Glodzik D, Zou X, Martincorena I, Alexandrov LB, Martin S, Wedge DC, Van Loo P, Ju YS, Smid M, et al. Landscape of somatic mutations in 560 breast cancer whole-genome sequences. Nature. 2016; 534:47-54. 